\title{
Is periodontitis associated with the severity of COVID-19?
}

\section{Erfan Shamsoddin ${ }^{1}$}

\section{A commentary on}

\section{Marouf N, Cai W, Said K N et al.}

Association between periodontitis and severity of COVID-19 infection: A case-control study. J Clin Periodontol 2021; 48: 483-491.

\section{Abstract}

Data sources The national electronic health records of the Hamad Medical Corporation (HMC) in the State of Qatar.

Data extraction and synthesis This case-control study included 568 patients from the HMC electronic database for data eliciting from 27 February 2020 to 31 July 2020. Patients with coronavirus disease 2019 (COVID-19) complications were considered as cases. Controls were defined as COVID-19 patients without major complications (discharged). Multivariate logistic regression was performed to assess the association between the exposures (periodontitis, demographics and medical conditions) and outcomes (COVID-19 complications). The association was adjusted for possible confounding factors. Additionally, sensitivity analyses were performed to account for stronger risk factors of the determined complications. The results were reported using the odds ratio (OR) and $95 \%$ confidence intervals $(\mathrm{Cls})$. Laboratory data (blood parameters concerning the course of COVID19) were also compared between the case and control groups. Results The patients were divided into 40 cases and 528 controls. Among the patients with periodontitis, $12.8 \%$ (33/258) went through COVID-19 complications, whereas $2.2 \%$ (7/310) of those without periodontitis experienced complications. The adjusted OR of patients with periodontitis was $3.67(95 \% \mathrm{Cl} 1.46-9.27)$ for all COVID-19 complications, 8.81 (95\% Cl 1.00-77.7) for death, $3.54(95 \% \mathrm{Cl}$ 1.39-9.05) for intensive care unit admission and 4.57 (95\% Cl 1.1917.4) for the need of assisted ventilation. After conducting subgroup analyses for age, diabetes and smoking, periodontitis was still significantly associated with all the outcomes. Laboratory biomarkers (haemoglobin A1c, white blood cells and C-reactive protein) had higher levels in COVID-19 patients with periodontitis than those without periodontal disease.

Conclusions This study concluded that periodontitis was significantly associated with higher risks of COVID-19 complications and higher blood marker levels. These results, however, are not of high quality and further research in this regard is required.

\section{Commentary}

Evaluating the association of dental disorders, especially periodontal diseases, with systemic conditions has always been a hot topic in dental sciences. Scrutinising such associations
Practice point

- It is possible that periodontal treatment might benefit COVID19 patients by reducing the severity of COVID-19 complications.

has become more salient with the emergence of the coronavirus disease 2019 (COVID-19) pandemic. This multicentre casecontrol study assessed the relationship between periodontitis and severity of infection in COVID-19 patients who attended the Hamad Medical Corporation (HMC) hospitals in Qatar. According to the inclusion criteria, from 1,076 patients identified with COVID-19 and ongoing dental records who attended the centres for nearly five months after 27 February 2020, 568 were included. All the cases (40 patients) and controls (528 patients) were under 18 years old and required dental radiographs to be included in the study. The diagnosis of COVID-19 was confirmed using the World Health Organisation interim guidelines and two subsequent positive polymerase chain reaction (PCR) tests. The main exposure, periodontitis, was diagnosed in the patient records using posterior bitewing and panoramic radiographs. Whichever of these showed greater extent of bone loss was used to determine the periodontitis diagnosis. Local factors were not considered, but periodontitis had to be diagnosed in at least two non-adjacent teeth. The authors described the inclusion and exclusion criteria clearly and applied them consistently across all the study participants. However, the use of $2 \mathrm{D}$ radiographs (bitewing and panoramic) alone to diagnose periodontitis is not recommended and thus diagnostic accuracy was compromised. ${ }^{1}$ Also, the authors only used one diagnostic parameter for periodontitis diagnosis, namely interdental bone loss, which further weakens the diagnostic accuracy. Additionally, prioritising the radiograph showing the greater amount of bone loss to reach a diagnosis for periodontitis certainly brings doubt to mind about the accuracy of the authors' interpretation and the prevalence of periodontitis in their target population.

A multivariate regression model was used to assess the association between periodontitis and COVID-19 complications. Laboratory values (blood markers) were also evaluated and compared between groups using the Mann-Whitney U test. The authors expected an R figure of 0.3 , a significance level of 0.05 and a minimum sample size of 320 (for regression model) to achieve an $80 \%$ statistical power. They adjusted the regression model for various risk factors of periodontitis and COVID-19 (that is, age, sex, diabetes, comorbidity and smoking behaviour). According to their findings, the adjusted odds ratios (ORs) of the regression analysis were as follows: 3.67 (95\% CI 1.46-9.27) for all COVID19 complications, 8.81 (95\% CI 1.00-77.7) for death, 3.54 (95\% CI 1.39-9.05) for intensive care unit admission and 4.57 (95\% 


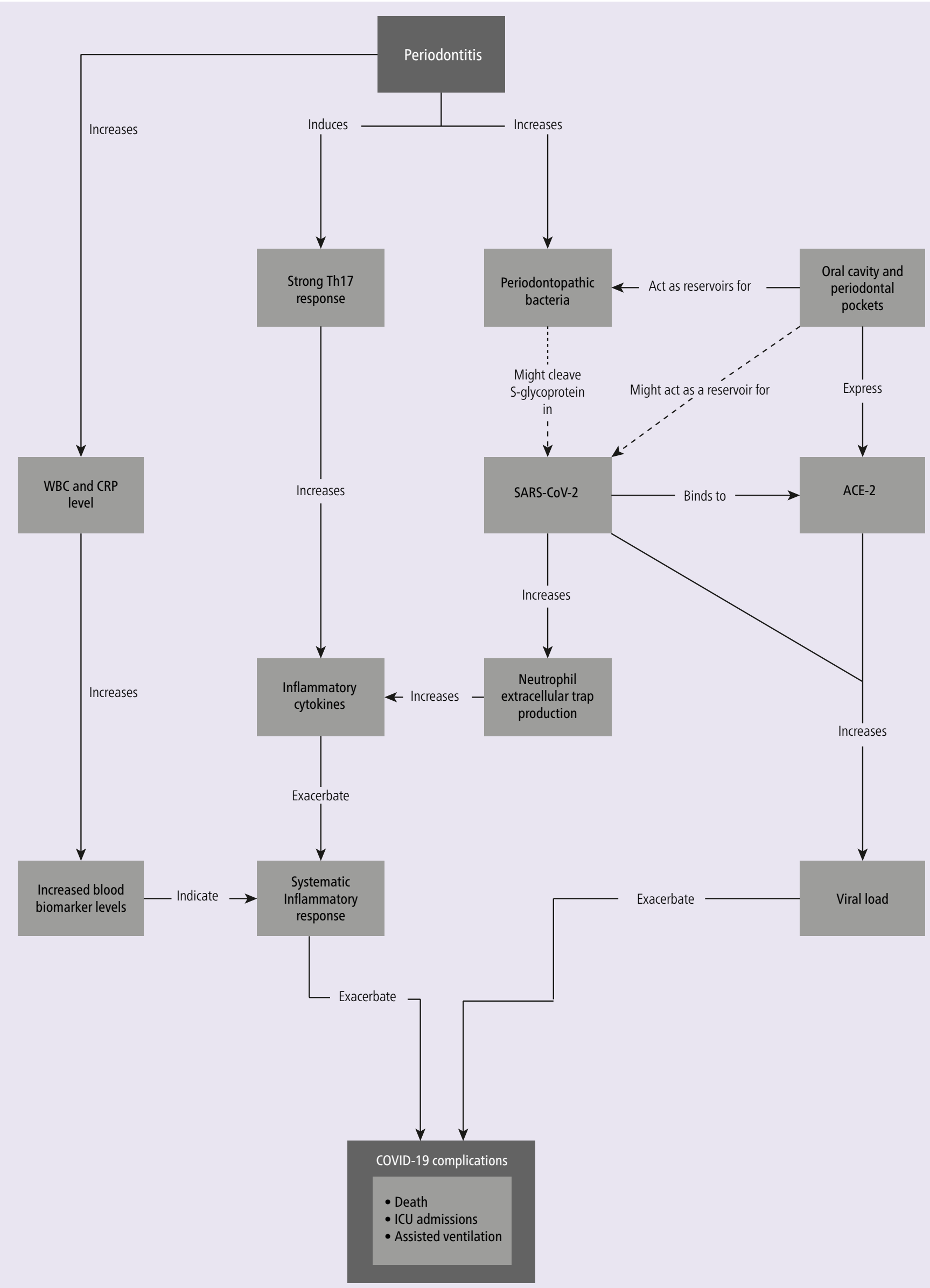

Fig. 1 The main pathways proposed for the association of periodontitis and COVID-19 severity 
CI 1.19-17.4) for the need of assisted ventilation. Inflammatory blood marker levels (for example, haemoglobin A1c, white blood cells and C-reactive protein) were significantly higher in COVID19 patients who had periodontitis than those who did not. The authors tried to cover a comprehensive range of comorbidities for the model adjustment, though did not consider a critical issue: the patients' socioeconomic status. They only considered age and sex as the demographic information and did an additional sensitivity analysis while stratifying the data based on age groups. This is a clear drawback in this study as the role of income and ethnicity in COVID-19 severity is already illuminated in the literature. ${ }^{2}$ It is very plausible that patients who were worse off in terms of income had waited longer to attend a public hospital to get the necessary care. Another issue is that the data was gathered from 14 centres, so there may have been access inequity for some patients. These factors could contribute to more severe COVID-19 complications, thus undermining the accuracy of model adjustments. ${ }^{3}$

Furthermore, the authors did not report R squared figures in the paper (neither the text nor the supporting information), so any reporting bias in their results was not identified. The expected R was also very low (0.3), which further suggests high variability and therefore inaccuracies in the findings of the study. The notable changes of ORs from the raw values to the adjusted ones for all outcomes (all COVID-19 complications, death, intensive care unit admission and assisted ventilation) strengthen this doubt about the primary exposure and probably indicate a spurious relationship.

The association of periodontitis with systemic diseases like nosocomial pneumonia has already been investigated. ${ }^{4}$ However, claiming strong and 'significant' associations between periodontitis and widespread diseases like COVID-19 is not substantiated by this study. Selective presentation of supportive evidence to suggest periodontal diseases as risk factors for the severity of COVID-19 might bring more damage than benefit to the health systems. ${ }^{5}$ The inaccuracy of dental studies in assessing the relationship between periodontal diseases and systemic conditions has already been stated. ${ }^{6}$

Be that as it may, periodontal diseases can increase the inflammatory response in patients, which might exacerbate the systemic symptoms and clinical course of COVID-19. Altering the expression of cellular receptors, enhancing the virulence of the SARS-CoV-2 virus and periodontal pockets acting as viral reservoirs are other mechanisms suggested to explain the role of periodontitis in COVID-19 severity. ${ }^{7}$ Figure 1 illustrates the concepts and theories stated by this paper for the association of periodontitis and the severity of COVID-19. On the other hand, considering the significant burden of COVID-19 and its high prevalence, it is of utmost importance to assess the association of diseases and comorbidities with COVID-19. Conducting more studies with a more rigorous methodology, less biased approach and broader scale (higher external validity and generalisability) will be necessary in order to establish the relationship between periodontitis and COVID-19 exists.

\section{Author affiliation}

${ }^{1}$ National Institute for Medical Research Development (NIMAD), Tehran, Iran.

Correspondence to: Erfan Shamsoddin

\section{Acknowledgements}

Thanks to Dr Ahmad Sofi-Mahmudi as his comments notably contributed to enhancing this paper.

\section{References}

1. Takeshita W M, Vessoni Iwaki L C, Da Silva M C, Tonin R H. Evaluation of diagnostic accuracy of conventional and digital periapical radiography, panoramic radiography, and cone-beam computed tomography in the assessment of alveolar bone loss. Contemp Clin Dent 2014; 5: 318-323.

2. Prats-Uribe A, Paredes R, Prieto-Alhambra D. Ethnicity, comorbidity, socioeconomic status, and their associations with COVID-19 infection in England: a cohort analysis of UK Biobank data. 2020. Available online at https://www.medrxiv.org/ content/10.1101/2020.05.06.20092676v3 (accessed May 2021)

3. Jamshaid H, Zahid F, Din I U et al. Diagnostic and Treatment Strategies for COVID19. AAPS PharmSciTech 2020; 21: 222.

4. Yang L C, Suen Y J, Wang Y H, Lin T C, Yu H C, Chang Y C. The Association of Periodontal Treatment and Decreased Pneumonia: A Nationwide Population-Based Cohort Study. Int J Environ Res Public Health 2020; 17: 356.

5. Taylor H, Rahurkar S, Treat T, Thyvalikakath T, Schleyer T. Does Nonsurgical Periodontal Treatment Improve Systemic Health? J Dent Res 2021; 100: 253-260.

6. Raittio E, Farmer J. Methodological Gaps in Studying the Oral-Systemic Disease Connection. J Dent Res 2021; 100: 445-447.

7. Takahashi Y, Watanabe N, Kamio N, Kobayashi R, linuma T, Imai K. Aspiration of periodontopathic bacteria due to poor oral hygiene potentially contributes to the aggravation of COVID-19. J Oral Sci 2020; 63: 1-3.

Evidence-Based Dentistry (2021) 22, 66-68.

https://doi.org/ 10.1038/s41432-021-0179-x 